\title{
ÄQUIVALENZRELATION \\ FÜR DIE CHARAKTERISIERUNG DES JACOBSONSCHEN RADIKALS
}

\author{
Von \\ F. SZÁSZ (Budapest)
}

Für eine universale Algebra $\mathfrak{Q}=(A, \Omega)$ im Sinne von $\mathrm{G}$. Birkhoff und für eine Teilmenge $B$ der Menge $A$ der Strukturelemente bezeichne $B_{\Omega}$ die durch $B$ erzeugte Teilstruktur. Enthält $B$ nur ein enziges Element $b$, so wird $B_{\Omega}$ mit $(b)_{\Omega}$ bezeichnet. Durch die Gleichung $(a)_{\Omega}=(b)_{\Omega}$ wird in $A$ eine Äquivalenzrelation definiert.

Ist nun insbesondere $\mathfrak{U}=(A, \Omega)$ ein assoziativer Ring, wobei $\Omega$ das System der Addition, Substraktion und aller Rechtsmultiplikationen bezeichnet, so stimmt $(a)_{\Omega}$ mit dem Hauptrechtsideal $(a)_{r}$ von $A$ überein, und dann ist die durch die Gleichung $(a)_{r}=(b)_{r}$ definierte Äquivalenzrelation $\equiv$ auch eine Linkskongruenz der multiplikativen Halbgruppe des Ringes $A$, die mit der Hilfe von ganzen rationalen Zahlen und von Ringelementen auch explizit beschrieben werden kann.

Das Ziel dieser Note ist, das Jacobsonsche Radikal $J$ eines Ringes $A$ (vgl. JACOBSON [3]) mit der Hilfe der obigen Äquivalenzrelation zu charakterisieren. Demnach läßt sich $J$ als ein einseitig antieinfaches Radikal ansehen (vgl. AnDRUNAKIEWITSCH [1]). Die im Beweis benützte Methode ist teilweise einem Beweis von SĄSIADA [5] und des Verfassers [6] ähnlich.

Es gilt nämlich der folgende

SATZ. Das Jacobsonsche Radikal $J$ eines Ringes A stimmt mit der Menge $K$ derjenigen Elemente $x$ von A überein, für welche die (im vorigen erklärte) Äquivalenzrelation $y \equiv y+y z$ mit jedem Element $y \in A$ und mit jedem Element $z$ des Hauptrechtsideals $(x)_{r}$ gilt.

BEWEIS. Bezeichnen $B^{-1} C$ für beliebige Teilmengen $B$ und $C$ des Ringes $A$ die Menge

$$
\{w ; w \in A, B w \subseteq C\}
$$

und $\Phi_{r}$ das Frattinische Rechtsideal (d.h. den Durchschnitt aller maximalen Rechtsideale) von $A$, so läßt sich nach Hille [2] bzw. Kertész [4] $J=A^{-1} \Phi_{r}$ bestätigen.

Zuerst beweisen wir $K \subseteq J$. Gilt nämlich $x \notin J$ für ein $x \in A$, so existieren wegen $x \in A^{-1} \Phi_{r}$ ein Element $y \in A$ und ein maximales Rechtsideal $R$ von $A$ mit $y x \in R$. Dann ergibt sich $A^{2} \nsubseteq R, y \notin R$ und der $A$-Rechtsmodul $A / R$ ist einfach. Folglich gibt es ein $u \in A$ mit $y+R=y x u+R$. Dann gilt wegen $z=-x u \in(x)_{r}$, und

gewiß $x \notin K$.

$$
(y+y(-x u))_{r} \leqq R \neq A=(y)_{r}+A
$$

Zweitens zeigen wir, daß auch $J \subseteq K$ gilt. Ist nämlich $x \notin K$ für ein $x \in A$, so existieren Elemente $z \in(x)_{r}$ und $y \in A$, derart, daß $y+y z$ und $y$ nicht äquivalent 
bezüglich $\equiv$ sind. Man erhält dann $(y)_{r} \neq(y+y z)_{r}$ und $(y)_{r} \supset(y+y z)_{r}$. Ist nun $\mathfrak{M}$ die Menge derjenigen Rechtsideale $R$ von $A$, für die

$$
(y+y z)_{r} \subseteq R \text { und } y \notin R
$$

gelten, so ist $\mathfrak{M}$ nichtleer und induktiv. Es sei $M$ ein maximales Rechtsideal aus $\mathfrak{M}$ (welches nach dem Zornschen Lemma existiert). Der $A$-Rechtsmodul $A / M$ ist dann subdirekt irreduzibel, denn $(y)_{r}+M / M$ liegt in jedem von Null verschiedenen $A$-Teilmodul von $\boldsymbol{A} / M$. Wegen $y \notin M$ und $y+y z \in M$ erhält man $(y z)_{r}+M=$ $=(y)_{r}+M$, weiterhin

$$
(y z)_{r}+M \sqsubseteq y(x)_{r}+M \neq M .
$$

Da $(y)_{r}+M / M$ ein einfacher $A$-Rechtsmodul ist, der von dem Radikal $J$ annihiliert wird, gilt $x \notin J$.

Hiernach gilt $J=K$, womit der Satz bewiesen ist.

Bemerkung (am 27. August 1971): Übungsaufgabe 5.24 des Lehrbuches ,,Vorlesungen über Artinsche Ringe" von A. KERTÉsz führt ein Radikal $K^{*}(M)$ für jeden $A$-Rechtsmodul $M$ über einem Ring $A$ ein. Nach dem Verfasser (Rings, which are radical modules; Proc. Japan Acad., im Erscheinen) ist $A$ dann und nur dann ein Jacobsonscher Radikalring, wenn $A$, als ein $A$-Rechtsmodul $A$ angesehen, ein Radikalmodul ist, d. h. $K^{*}(A)=A$ gilt. Andererseits hat der Verfasser gezeigt (Notes on modules, III; Proc. Japan Acad. 46:3 (1970) 354-357), dass $K^{*}(A)$ klein Radikal, im Sinne von S. A. Amitsur und A. G. Kurosch, des Ringes $A$ ist.

(Eingegangen am 28. Mai 1969, bearbeitet am 30. Januar 1970)

MAGYAR TUDOMÁNYOS AKADÉMTA

MATE MATIKAI KUTATÓ INTÉZETE

BUDAPEST, V., REÁLTANODA U. $13-15$.

\section{Literaturverzeichnis}

[1] W. A. Andrunakiewitsch, Antieinfache und streng idempotente Ringe, Isv. Akad. Nauk SSSR. Ser. Math. 21 (1957) S. 125-144. (Russisch)

[2] E. Hille, Functional Analysis and Semi-Groups (Providence, 1948) Amer. Math. Soc. Colloq, Publ., vol. 31.

[3] N. Jacobson, Structure of Rings (Providence, 1964, 2, edition), Amer. Math. Soc. Colloq. Publ., vol. 37.

[4] A. Kertész, A characterization of the Jacobson radical, Proc. Amer. Math. Soc. 14:4 (1963), S. 595-597.

[5] E. SAzStada, Solution of the problem of existence of simple radical rings, Bull. Acad. Polon. Sci. Klasse III, 9 (1961), S. 257.

[6] F. SzÁsz, Bemerkungen über Rechtssockel und Nilringe, Monatshefte f. Math. 67 (1963), S. $359-362$. 